\title{
A simplified laparoscopic ventral hernia repair: the scroll technique
}

\author{
A. Hussain · S. El-Hasani
}

Published online: 27 February 2009

(C) Springer Science+Business Media, LLC 2009

\section{Dear Sir,}

We read with great interest the article entitled A Simplified Laparoscopic Ventral Hernia Repair: The Scroll Technique published in Surgical Endoscopy [1]. We congratulate authors for their excellent results and their description of a new technique for mesh insertion in the laparoscopic repair of ventral hernia.

With our technique, published in the JSLS [2], we introduce the mesh through a trocar placed in the center of the hernia defect itself. This provides two benefits. It prevents trocar-site incisional hernia, reported in other series [3], and avoids inducing weakness in other sites of the abdomen and a difficult closure, especially in obese patients. The orientation and placement of the mesh is ensured by putting a long suture in the center of the mesh and two stitches in the mesh at the 6 and 12 o'clock positions before insertion. The end of the long suture remains outside the abdomen through the trocar when the mesh is fully inside the abdomen. Pulling on that long suture helps to centralize the mesh in relation to the hernia defect. The two sutures in the 6 and 12 o'clock positions help to orient the mesh in longitudinal or transverse position according to the shape and size of the hernia defect.

The authors reported one case of mesh infection, and we are interested in the way this problem has been managed. Also, they reported no case of incisional hernia at the 12-mm trocar site, and we are interested in knowing whether this issue was investigated during the follow-up period.

Another point is seroma formation associated with polytetrafluoroethylene (PTFE). The authors explained this as due either to the tight weaving of the mesh or to the mesh material. They also recommend abdominal binder postoperatively to reduce the seroma formation.

In our practice, we did not find a significant association between the type of mesh and seroma formation, which we believe is entirely related to the size of the hernia, the thickness of subcutaneous fat, extent of dissection and the hemo-static technique and not to the type of mesh. Having said that, we also advise abdominal binder postoperatively for 2 weeks because we believe this will reduce the seroma formation.

\section{References}

1. Saber AA, Elgamal MH, Rao AJ, Itawi EA, Mancl TB (2008) A simplified laparoscopic ventral hernia repair: the scroll technique. Surg Endosc 22:2527-2531

2. Hussain A, Mahmood H, Shuaib S, El-Hasani S (2008) Prevention of trocar-site incisional hernia following laparoscopic ventral hernia repair. JSLS 12:206-209

3. Tonouchi H, Ohmori Y, Kobayashi M, Kusunoki M (2004) Trocarsite hernia. Arch Surg 139:1248-1256 